\title{
Modelling of Oil Film Bearings
}

\author{
Tatsuro Ishibashi ${ }^{1}$ Tadao Kawai ${ }^{2}$ \\ ${ }^{1}$ Meidensha Corporation, Japan, ishibashi-tat@mb. meidensha . co.jp \\ ${ }^{2}$ Department of Mechanical \& Physical Engineering, Osaka City University, Japan, \\ kawai@mech.eng.osaka-cu.ac.jp
}

\begin{abstract}
In this paper, we model the oil film bearings and estimate the fluid-induced instability for the design and the diagnosis of the rotating machinery system. The presented model is implemented in our original rotating machinery library by Modelica. An example of a Jeffcott rotor system supported by plain circular journal bearings is simulated. To check the behavior of the model, Campbell diagrams and stability maps are computed by using the Modelica_LinearSystems2 library.
\end{abstract}

Keywords: Rotor Dynamics, Oil Whirl, Oil Whip, Campbell Diagram, Stability Map

\section{Introduction}

Oil film bearings are widely used for the large rotating machinery systems such as turbines and generators. The oil film bearing has the following advantages compared to the rolling bearing. The oil film bearings provide the higher damping, which is required to pass through a critical speed and suppress vibration. Those also reduce noise, and have very long life under normal load condition because of the lack of contact between rotating parts. In harsh operating conditions such as high load and moderately high speed rotation, the oil film bearing has a superior performance. Some disadvantages of the oil film bearing are the higher friction, the higher susceptibility to particulate contamination and that it cannot run without a lube system.

At high rotating speed, self-excited vibration due to the motion of the oil film may occur in the rotating machinery system supported by oil film bearings. This instable vibration, generally called oil whirl or whip causes damage to the machine. To design the high rotating speed machinery, it is necessary to understand this instable vibration mechanism and prevent it. To diagnose the rotating machinery system supported by oil film bearings, it is important to grasp the behavior with faults such as unbalance and shaft bending etc.

In this paper, the oil lubricated plain journal bearing model is implemented in our original Rotating Machinery library (Ishibashi et al, 2017). We simulate the rotating machinery system supported by oil film bearings with static unbalance in a rotor. The components in this system are from our original rotating machinery library based on transfer matrix method. To check the behavior of the model, Campbell diagrams and stability maps are computed by using the Modelica_LinearSystems2 library.

\section{Hydrodynamic lubrication}

In this Section, we derive the Reynolds equation in order to determine the force that the oil film exerts on the shaft, that is, the pressure distribution of the oil film (Hori and Kato, 2008; Ishida and Yamamoto, 2012; Matsushita et al, 2017). Figure 1 schematically shows both walls of the shaft and the bearing are separated by an oil film.

By treating the oil film as a continuum, the equation of motion of the oil film in three directions is derived from the Navier-Stokes equation. The majority of oil lubricated journal bearings operate in the slow viscous flow regime where the viscous forces are much greater than the inertia forces. In most of the industrial application, the bearing Reynolds number is usually below 1,500. Here, by simplifying various assumptions (rigid wall, incompressible Newtonian fluid, isothermal, isoviscous and isopycnic fluid, laminar flow, superior of differential coefficient in oil film thickness direction and ignoring inertial forces), equations of motion in the $x, y$ and $z$ directions are expressed as follows at any coordinate $(x, y, z)$.

$$
\begin{gathered}
\mu \frac{\partial u^{2}}{\partial y^{2}}=\frac{\partial p}{\partial x} \\
\mu \frac{\partial v^{2}}{\partial y^{2}}=0 \\
\mu \frac{\partial w^{2}}{\partial y^{2}}=\frac{\partial p}{\partial z}
\end{gathered}
$$

Here, $\mu$ is the uniform fluid viscosity of the lubricating oil, $u, v, w$ are the oil film flow velocity in the $x, y, z$ direction, respectively. The pressure generated in the oil film varies depending on the in-plane coordinate $x, z$, but it is uniform in the $y$ direction. By assuming that the oil film flow velocity on the wall surface is equal to the velocity of the wall surface motion, the boundary conditions are the velocities on the wall surfaces respectively. By integrating Equations 1, 2 and 3, the flow velocity is obtained as follows. 


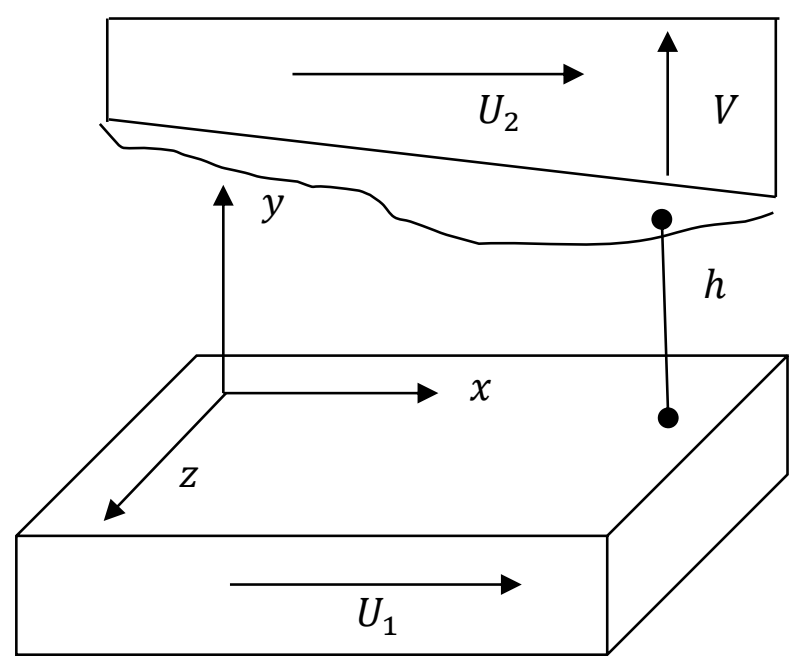

Figure 1. Lubrication surface model. $x$ is the journal rotating direction, $y$ is the oil film thickness direction, and $z$ is the bearing width direction. The oil film thickness $h$ does not change in the $z$ direction, but narrows down gradually in the $x$ direction. On the bearing surface $y=0$, the oil film moves at velocity $U_{l}$ in $x$ direction. On the journal surface $y=h$, it moves at velocity $U_{2}$ in $x$ direction. And it moves at velocity $V$ in $y$ direction.

$$
\begin{gathered}
u=\left\{U_{2}+\left(U_{1}-U_{2}\right) \frac{h-y}{h}\right\}+\left\{-\frac{y(h-y)}{2 \mu} \frac{\partial p}{\partial x}\right\} \\
v=V \frac{y}{h} \\
w=-\frac{y(h-y)}{2 \mu} \frac{\partial p}{\partial z}
\end{gathered}
$$

These flow velocity equations are substituted into the equation obtained by integrating the following continuous Equation 7 once for $y$.

$$
\frac{\partial u}{\partial x}+\frac{\partial v}{\partial y}+\frac{\partial w}{\partial z}=0
$$

A formula for converting the order of differentiation and integration is applied with respect to terms of $x$ derivative and $z$ derivative of the pressure $p$,

$$
\int_{0}^{h(x)} \frac{\partial}{\partial x} f(y, x) d y=\frac{\partial}{\partial x} \int_{0}^{h(x)} f(y, x) d y-f(h(x), x) \frac{\partial h(x)}{\partial x}
$$

A three-dimensional dynamic Reynolds equation is obtained as follows.

$$
\begin{gathered}
\frac{\partial}{\partial x}\left(h^{3} \frac{\partial p}{\partial x}\right)+\frac{\partial}{\partial z}\left(h^{3} \frac{\partial p}{\partial z}\right) \\
=6 \mu\left(U_{1}-U_{2}\right) \frac{\partial h}{\partial x}+6 \mu h \frac{\partial}{\partial x}\left(U_{1}+U_{2}\right)+12 \mu V
\end{gathered}
$$

\section{Static property of oil film bearing}

Equation 9 is adapted to the plain circular journal bearing which have the radius $R$, the width $L$ and the radial clearance $C$ shown in Figure 2. The uniform fluid viscosity $\mu$, the constant journal circumferential velocity $U$ and the constant bearing load $W$ are assumed.

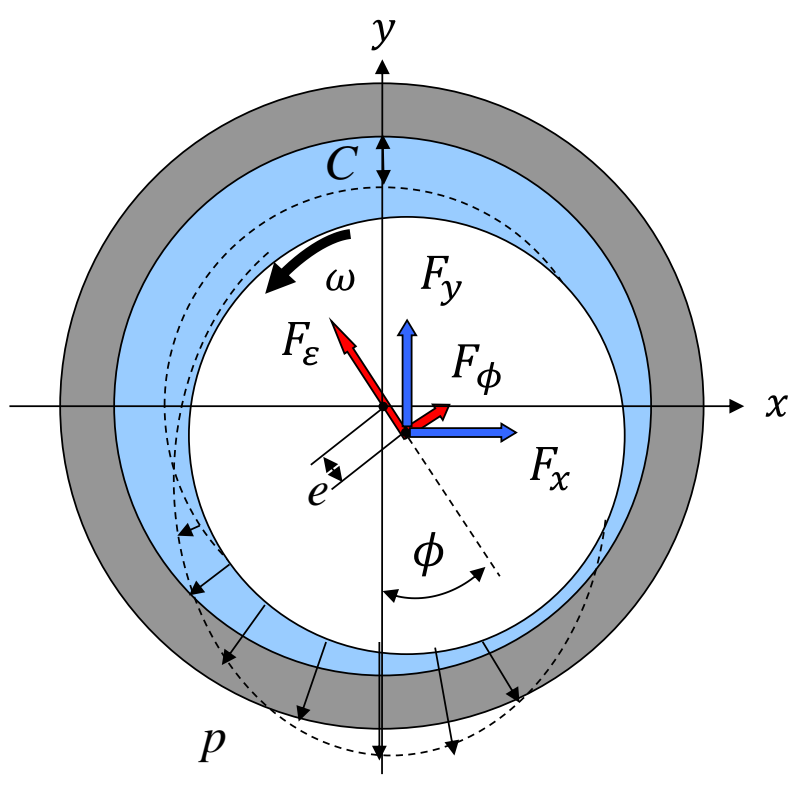

Figure 2. Oil film force and pressure of the plain circular journal bearing.

Substituting $U_{1}=0, U_{2}=U, V=U \frac{\partial h}{\partial x}+\frac{\partial h}{\partial t} \quad$ into Equation 9 and applying the short bearing approximation $\frac{\partial p}{\partial x} \ll \frac{\partial p}{\partial z}$, Equation 9 can be calculated as follows.

$$
h^{3} \frac{\partial^{2} p}{\partial z^{2}}=6 \mu U \frac{\partial h}{\partial x}+12 \mu \frac{\partial h}{\partial t}
$$

As the actual $C / R$ is very small value, the oil film thickness $h$ is approximately expressed as follows.

$$
h=C(1+\varepsilon \cos \phi)
$$

$\varepsilon$ is the eccentricity ratio $e / C$, where $e$ is the distance between the bearing and the journal center, $\phi$ is the journal center angle from the maximum clearance position for the direction of the journal rotation. Here, $x=R \phi$. Equation 10 is expressed by the following equation.

$$
\frac{d^{2} p}{d z^{2}}=-\frac{6 \mu}{C^{2}} \frac{\varepsilon \sin \phi}{(1+\varepsilon \cos \phi)^{3}}(\omega-2 \dot{\phi})+\frac{12 \mu}{C^{2}} \frac{\dot{\varepsilon} \cos \phi}{(1+\varepsilon \cos \phi)^{3}}
$$

Integrated twice under the boundary condition $p=0$ at the bearing edge $(z=0, L)$ with respect to $z$.

$$
p=\left(\frac{3 \mu}{C^{2}} \frac{\varepsilon \sin \phi}{(1+\varepsilon \cos \phi)^{3}}(\omega-2 \dot{\phi})-\frac{6 \mu}{C^{2}} \frac{\dot{\varepsilon} \cos \phi}{(1+\varepsilon \cos \phi)^{3}}\right)
$$

The oil film force is calculated by multiplying the oil film pressure by $-\cos \phi$ and $\sin \phi$, applying Gumbel's boundary condition and integrating in the bearing area $\theta=0 \sim \pi, z=0 \sim L$.

$$
\begin{aligned}
& F_{\varepsilon}=\int_{0}^{L} \int_{0}^{\pi}(-p \cos \phi) R d \phi d z \\
& =\frac{\mu R L^{3}}{C^{2}}\left(\frac{\varepsilon^{2}(\omega-2 \dot{\phi})}{\left(1-\varepsilon^{2}\right)^{2}}+\frac{\pi \dot{\varepsilon}\left(1+2 \varepsilon^{2}\right)}{2\left(1-\varepsilon^{2}\right)^{5 / 2}}\right)
\end{aligned}
$$




$$
\begin{aligned}
& F_{\theta}=\int_{0}^{L} \int_{0}^{\pi}(p \sin \phi) R d \phi d z \\
& =\frac{\mu R L^{3}}{C^{2}}\left(\frac{\pi \varepsilon(\omega-2 \dot{\phi})}{4\left(1-\varepsilon^{2}\right)^{3 / 2}}+\frac{2 \varepsilon \dot{\varepsilon}}{\left(1-\varepsilon^{2}\right)^{2}}\right)
\end{aligned}
$$

For the static equilibrium position $\dot{\varepsilon}=\dot{\phi}=0$, the oil film force is calculated as follows,

$$
\begin{gathered}
F_{\varepsilon_{0}}=\frac{\mu R L^{3}}{C^{2}}\left(\frac{\varepsilon_{0}^{2} \omega}{\left(1-\varepsilon_{0}^{2}\right)^{2}}\right) \\
F_{\phi_{0}}=\frac{\mu R L^{3}}{C^{2}}\left(\frac{\pi \varepsilon_{0} \omega}{4\left(1-\varepsilon_{0}^{2}\right)^{3 / 2}}\right)
\end{gathered}
$$

The eccentricity angle at the static equilibrium position is determined as follows.

$$
\tan \phi_{0}=\frac{F_{\phi_{0}}}{F_{\varepsilon_{0}}}=\frac{\pi \sqrt{1-\varepsilon_{0}^{2}}}{4 \varepsilon_{0}}
$$

The oil film force $x, y$ direction is written as follows

$$
\begin{gathered}
F_{x}=-F_{\varepsilon_{0}} \sin \phi_{0}+F_{\phi_{0}} \cos \phi_{0} \\
F_{y}=F_{\varepsilon_{0}} \cos \phi_{0}+F_{\phi_{0}} \sin \phi_{0}
\end{gathered}
$$

At the static equilibrium position, the oil film force balances with static load $W$. Hence $\varepsilon_{0}$ is written as follows.

$$
S\left(\frac{L}{2 R}\right)^{2}=\frac{\left(1-\varepsilon_{0}^{2}\right)^{2}}{\pi \varepsilon_{0} \sqrt{\pi^{2}+\left(16-\pi^{2}\right) \varepsilon_{0}^{2}}}
$$

Here, $S$ is Sommerfeld number written as follows.

$$
S=\frac{2 \mu N R L}{W}\left(\frac{R}{C}\right)^{2}
$$

Here, $N$ is the shaft rotating speed (rps, $1 / \mathrm{s})$. When $S\left(\frac{L}{2 R}\right)^{2}$ is given, the static equilibrium position $\varepsilon_{0}, \phi_{0}$ is uniquely determined.

\section{Dynamic property of oil film bearing}

Next we consider the dynamic property. The oil film force is highly nonlinear to solve. In order to discuss the linear stability of a shaft, the oil film force is linearized beforehand in the neighborhood of the static equilibrium position of the journal center. Further, in order to consider the journal motion in the rectangular coordinates system $(x, y)$ shown in the same figure, let us transform the polar components $F_{\varepsilon}$ and $F_{\phi}$ of the oil film force to the rectangular components $F_{x}$ and $F_{y}$. Then the oil film forces $F_{x}$ and $F_{y}$ can be written in the following form.

$$
\left[\begin{array}{l}
F_{x} \\
F_{y}
\end{array}\right]=\left[\begin{array}{ll}
k_{x x} & k_{x y} \\
k_{y x} & k_{y y}
\end{array}\right]\left[\begin{array}{l}
x \\
y
\end{array}\right]+\left[\begin{array}{ll}
c_{x x} & c_{x y} \\
c_{y x} & c_{y y}
\end{array}\right]\left[\begin{array}{l}
\dot{x} \\
\dot{y}
\end{array}\right]
$$

The oil film force is expressed by the four spring coefficients and the four damping coefficients. Each coefficient is made dimensionless by converting as follows.
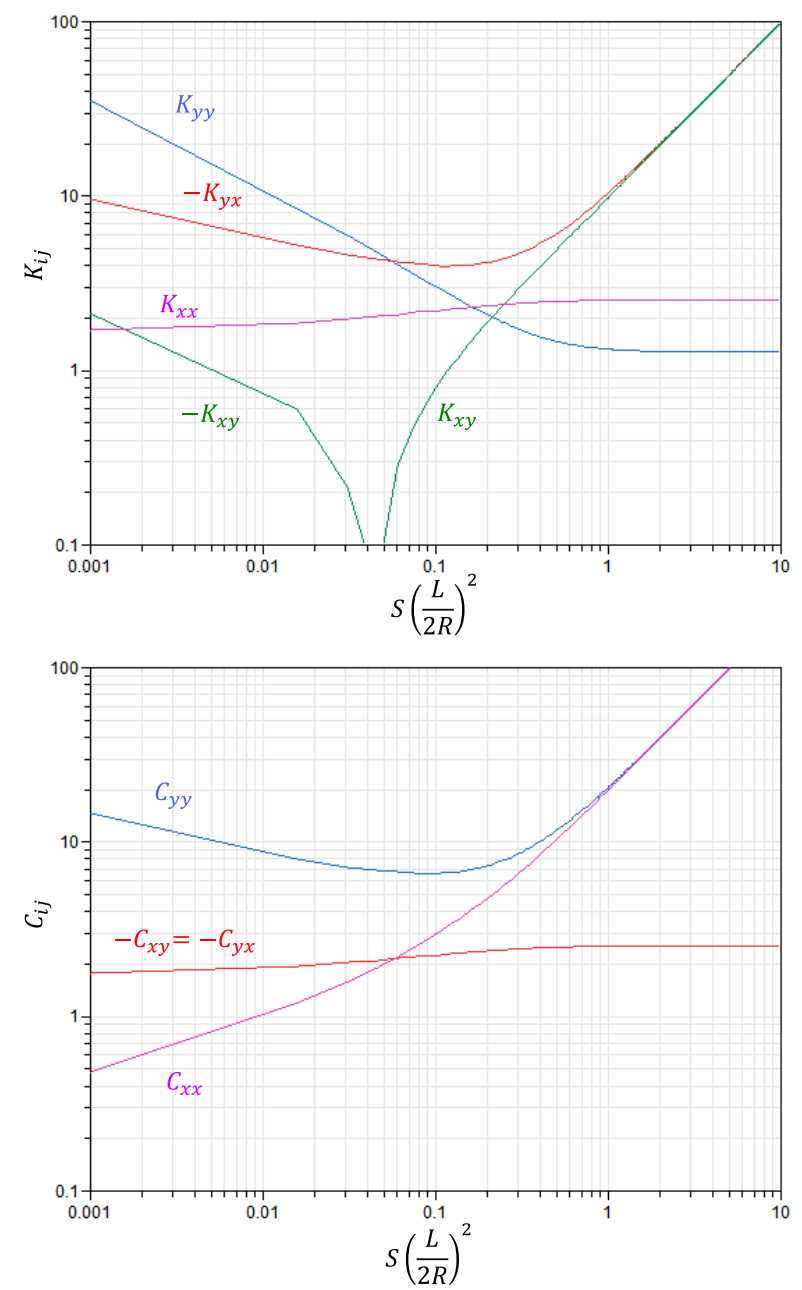

Figure 3. The dimensionless coefficients of the plain circular journal bearing in case of the short bearing approximation. Spring coefficients (upper). Damping coefficients (lower).

$$
\begin{aligned}
K_{i j} & =\frac{C}{W} k_{i j} \\
C_{i j} & =\frac{C \Omega}{W} c_{i j}
\end{aligned}
$$

For example, the dimensionless coefficients of the plain circular journal bearing in case of the short bearing approximation and Gumbel's boundary conditions are calculated as follows.

$$
\begin{gathered}
K_{x x}=\frac{4\left[2 \pi^{2}+\left(16-\pi^{2}\right) \varepsilon_{0}^{2}\right]}{K_{\alpha}} \\
K_{x y}=\frac{\pi\left[\pi^{2}-2 \pi^{2} \varepsilon_{0}^{2}-\left(16-\pi^{2}\right) \varepsilon_{0}^{4}\right]}{\varepsilon_{0}\left(1-\varepsilon_{0}^{2}\right)^{0.5} K_{\alpha}} \\
K_{y x}=-\frac{\pi\left[\pi^{2}+\left(32+\pi^{2}\right) \varepsilon_{0}^{2}+2\left(16-\pi^{2}\right) \varepsilon_{0}^{4}\right]}{\varepsilon_{0}\left(1-\varepsilon_{0}^{2}\right)^{0.5} K_{\alpha}} \\
K_{y y}=\frac{4\left[\pi^{2}+\left(32+\pi^{2}\right) \varepsilon_{0}+2\left(16-\pi^{2}\right) \varepsilon_{0}^{4}\right]}{\left(1-\varepsilon_{0}^{2}\right) K_{\alpha}} \\
C_{x x}=\frac{2 \pi\left(1-\varepsilon_{0}^{2}\right)^{0.5}\left[\pi^{2}-2\left(8-\pi^{2}\right) \varepsilon_{0}^{2}\right]}{\varepsilon_{0} K_{\alpha}}
\end{gathered}
$$




$$
\begin{gathered}
C_{x y}=C_{y x}=-\frac{8\left[\pi^{2}-2\left(8-\pi^{2}\right) \varepsilon_{0}^{2}\right]}{K_{\alpha}} \\
C_{y y}=\frac{2 \pi\left[\pi^{2}+2\left(24-\pi^{2}\right) \varepsilon_{0}^{2}+\pi^{2} \varepsilon_{0}^{4}\right]}{\varepsilon_{0}\left(1-\varepsilon_{0}^{2}\right)^{0.5} K_{\alpha}} \\
K_{\alpha}=\left\{\pi^{2}+\left(16-\pi^{2}\right) \varepsilon_{0}^{2}\right\}^{1.5}
\end{gathered}
$$

The dimensionless coefficients are expressed as the functions of the eccentricity $\varepsilon_{0}$ at the static equilibrium position. Therefore, as the static equilibrium position changes, the dimensionless coefficients change. In Figure 3, we plot the dimensionless coefficients against $S\left(\frac{L}{2 R}\right)^{2}$, which uniquely detemines the static equilibrium position $\varepsilon_{0}, \phi_{0}$.

The dimensionless coefficients of the other type of journal bearings are obtained by computing Reynolds equation with the specific boundary condition or referring to the database (Someya, 1989).

\section{Modelica Implementation}

The presented oil film force models must be supplied by constraints in the transverse direction $x, y$ and rotating angle direction. Our original Rotating Machinery library is used to supply these constraints (Ishibashi et al, 2017). The basic flange of this library has 5 DOF (degree of freedom), consisting of 4 DOF (two dimensional deflections and slopes) for transverse vibration of the rotor system and 1 DOF (rotating angle) for torsional vibration, neglecting axial vibration. Features like unbalanced rotors, flexible beams (shaft), supports, springs and dampers are all represented. The library is used to create the total rotating machinery system. The above static and dynamic of the oil film force models are implemented respectively.

The oil film force models are implemented with two connectors, each with 5 DOF. Since the above oil film force models has the only $3 \mathrm{DOF}$, the moments are set as zero. These connectors are the connections to the journal in the bearing (3 connectors rotor without unbalance) and the support.

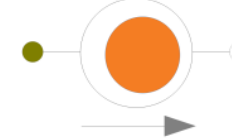

Figure 4. Modelica Icon of the oil film force.

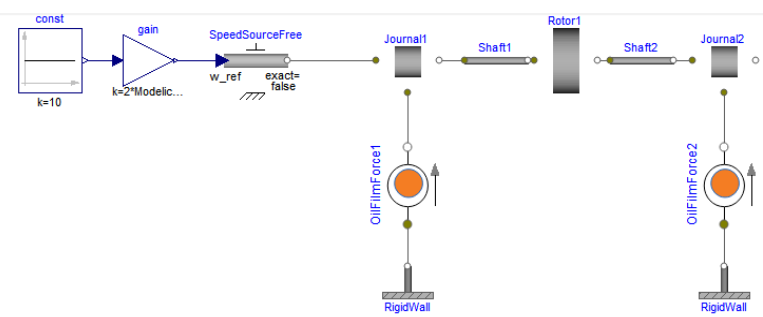

Figure 5. The rotating machinery system supported by the oil film bearing.
In Figure 4 the icon of the oil film force model is shown. The static and dynamic models have the same icon. No inertias or constraints are included in the model. Using our original rotating machinery library, it is possible to create rotating machinery systems. A simple rotating machinery system supported by the plain circular journal bearing is easily generated. Here, we treat Jeffcott rotor system as a test case in Figure 5. In the models, the static and dynamic oil film force models (Figure 4) are defined as described in this paper, all other components are components of our rotating machinery library.

\section{Simulation Results}

\subsection{Static property}

A static equilibrium position of a simple rotor machinery system supported by the oil film bearing (as shown in Figure 5) is estimated. Here, the oil film bearing is the plain circular journal bearing explained in the above section. By replacing the constant input with the ramp input and simulating the static model (in which rotors and shafts have only the loads of weights), the position of the Journall flange connected to the oil film force model i.e. the static equilibrium position is calculated. Also, the static equilibrium position is the same as the journal center. Figure 6 shows the trajectory of the journal center when the rotating speed increases. In the plain circular journal bearing, the static equilibrium position draws the trajectory close to semicircular arc shape. As the journal rotating speed increases infinitely, the journal center reaches the bearing center position.

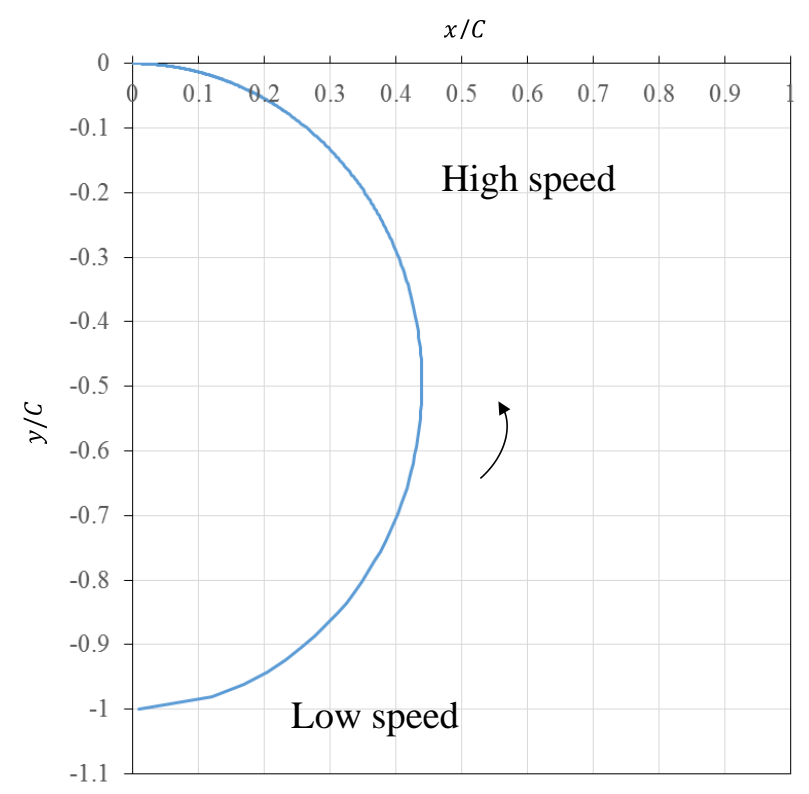

Figure 6. The static equilibrium position. 


\subsection{Dynamic property}

At the constant rotating speed, the static equilibrium position and the linearized dynamic oil film forces are uniquely determined. Using the model in Figure 5, the dynamic property of the journal center around the static equilibrium position is computed. The model has just a static unbalance in Rotor1. This system has the critical
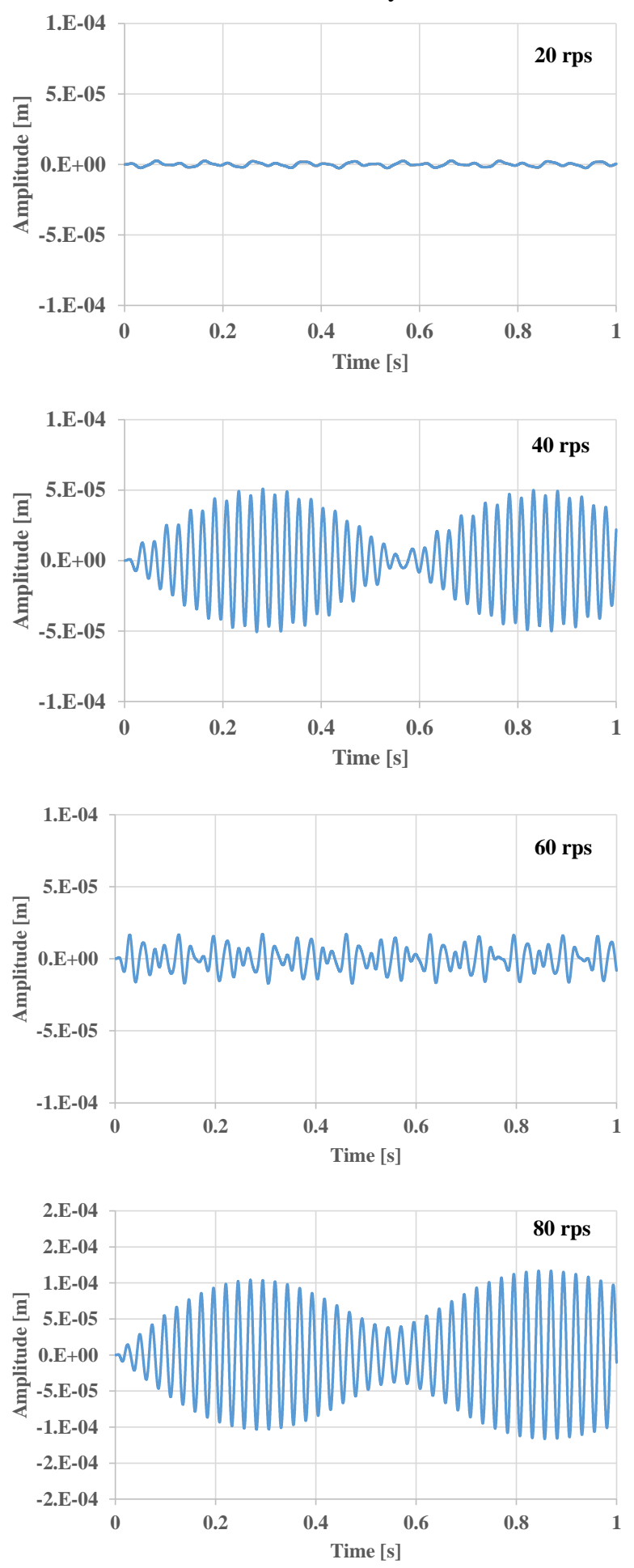

Figure 7. The simulation results of the journal center vibration at the constant rotating speed in $x$ direction. This system is the light shaft system. speed (the natural frequency of lateral vibration) around $42 \mathrm{~Hz}$ (see Figure 8). Figure 7 shows the transient simulation results of the journal center position in $x$ direction at the different constant rotating speed. At 80rps, the vibration gradually diverges. This implies the instable vibration. To analyze these simulation results and the dependency of the rotating speed, these simulation results are processed by FFT (Fast Fourier Transformation). The waterfall plot is created as shown in Figure 8 (a). In the light shaft system such as Figure 8 (a), the vibration of the half rotating speed increases as the rotating speed increases. When the rotating speed reaches twice the critical speed, a large whirl occurs. Because the whirling speed of the half-speed whirl coincides with the natural frequency of the system. Beyond this point, a large whirling still continues.

To check the oil film bearing response, we simulate the model by increasing the constant bearing load $W$. Figure 8 (b) shows the result. Below the critical speed, the halfspeed whirl is not observed. The static unbalance vibration synchronized with the rotating speed is observed. Beyond the critical speed, the large vibration of the natural frequency is still observed.
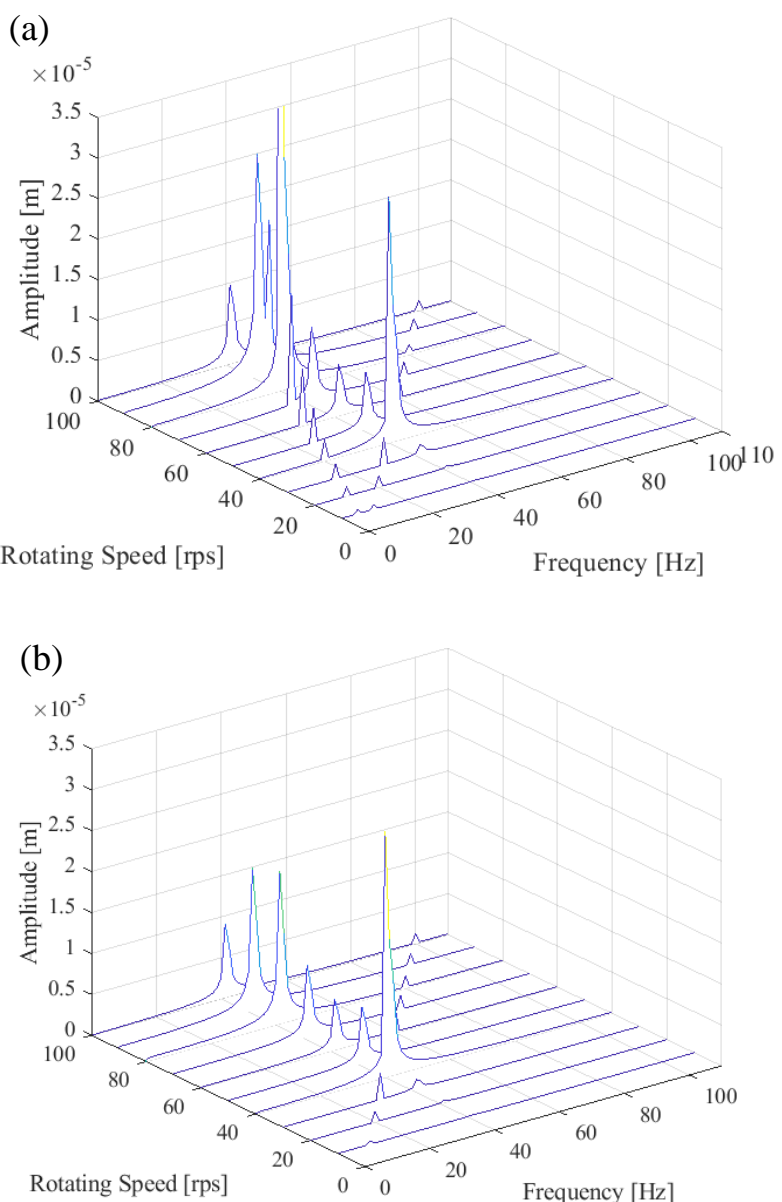

Figure 8. Waterfall plots of the simulation results.

(a) Light shaft. (b) Heavy shaft. 


\subsection{Eigenfrequency and Stability Analysis}

Using the Modelica_LinearSystems2 library (Bauer $e t$ $a l, 2009$; Otter, 2006) and creating the functions, it is possible to create Campbell diagrams (also known as whirl speed maps) and stability maps, which show variations of eigenfrequencies and damping ratios of rotors with respect to the rotating speed. The models are linearized into the state space and represented in $\mathrm{ABCD}$ matrices by the Modelica_LinearSystems2 library. Computing eigenvalues of the matrix $\mathrm{A}$ and transforming into eigenfrequencies and damping ratios at the constant rotating speed, Campbell diagrams and stability maps are obtained. In rotating machinery systems, the eigenfrequencies often depend on the rotating speeds due to the induced gyroscopic effects or variable hydrodynamic conditions in fluid bearings. The intersection between the synchronous excitation line and the eigenfrequencies in Campbell diagrams are referred as critical speeds. In fluid bearing, damping ratios turn negative from positive as the rotating speed increases in stability maps. This speed is known as the instability threshold. Self-excited instability occurs at the speeds above the instability threshold. It is very
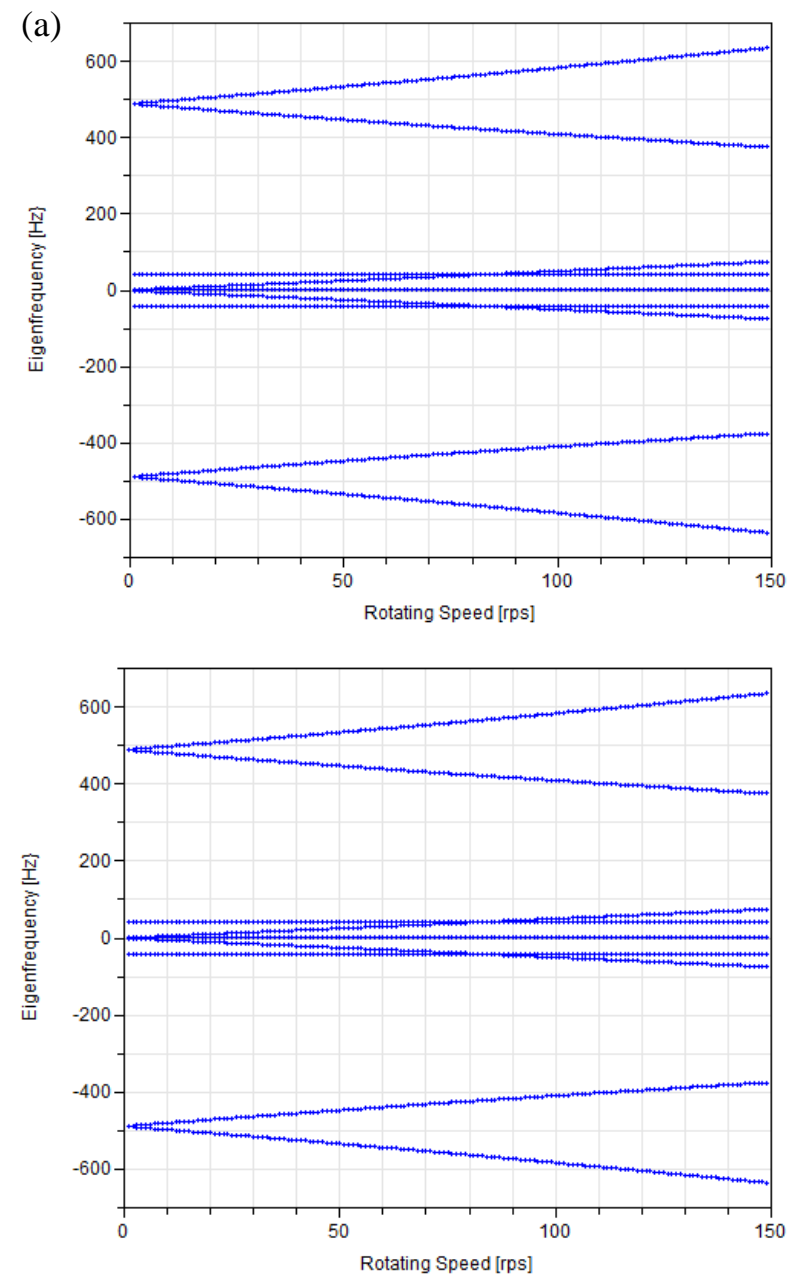

Figure 9. Campbell diagrams. (a) Light shaft. (b) Heavy shaft. important for design and diagnose the rotating machinery systems to compute analytically and measure experimentally critical speeds and instability thresholds. Here, we analyze the model corresponding to Figure 8. Figure 9 shows the Campbell diagrams. The Campbell diagrams show both forward and backward vibration modes. The Campbell diagrams of both of the light and heavy shaft show the totally same behavior. The critical frequency is around $42 \mathrm{~Hz}$, at which frequency the lateral vibration occurs. The eigenfrequency curves starting from around $500 \mathrm{~Hz}$ split due to the gyroscopic effects. Since the model only have a static unbalance, the vibration amplitude is too small to observe. The halfspeed whirl is observed due to the oil film.

Figure 10 shows the stability map. There is differences in the damping ratio curves between the light shaft and the heavy shaft. In the light shaft system, the damping ratio turns negative below the critical speed. From this speed the instable self-excited vibration called oil whirl occurs. The damping ratio reaches minimum at twice the critical speed. Over twice the critical speed, it increases. The oil whirl develops to the large whirl called oil whip at twice the critical speed and shrinks over twice the critical speed.
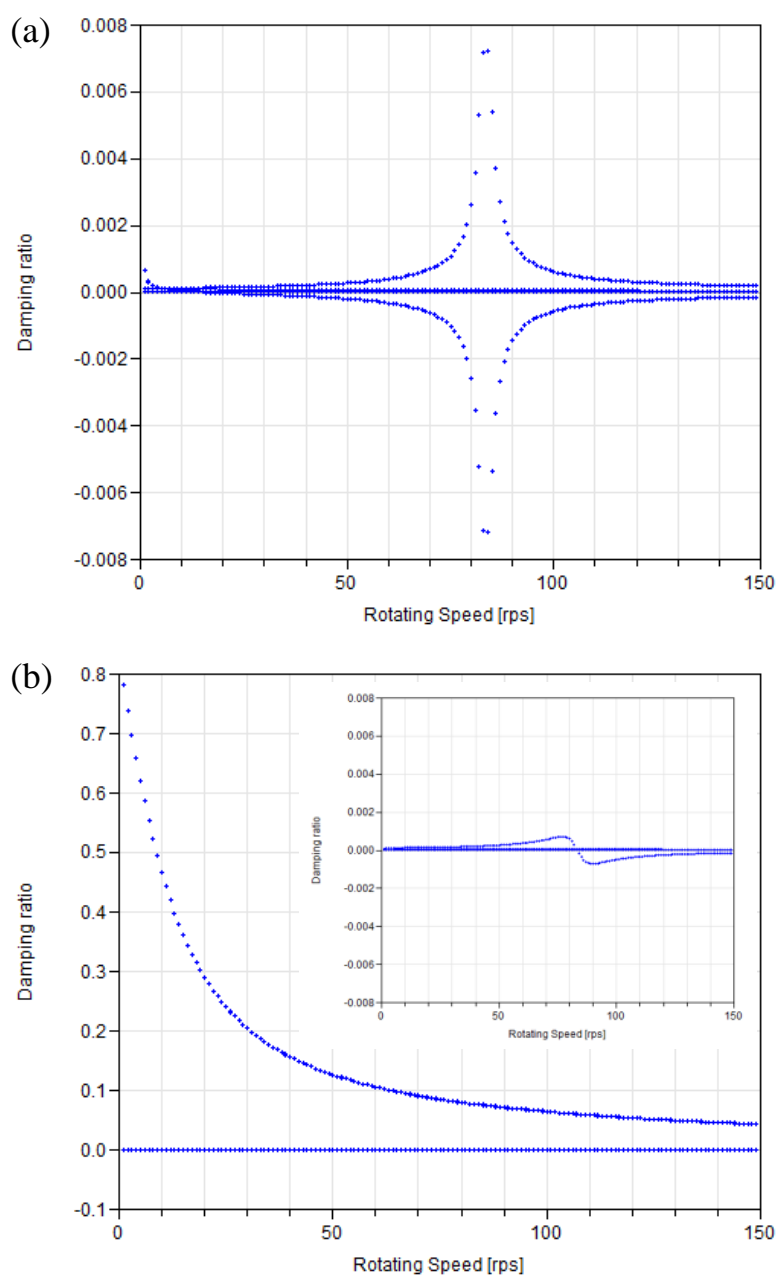

Figure 10. Stability maps. (a) Light shaft. (b) Heavy shaft. The inset shows the enlarged view. 
In the heavy shaft system, the damping ratio turns negative just below twice the critical speed. In the light shaft system, the damping ratios are smaller than in the heavy shaft. This means the light shaft system is more instable. These behaviors are well consistent with Figure 8.

\section{Conclusion}

In this paper, models are presented to describe the oil film force. Using our original rotating machinery library, it is possible to model a rotating machinery system supported by an oil film bearing. An example of Jeffcott rotor system supported by plain circular journal bearings is simulated and analyzed. The presented models make it possible to estimate Campbell diagrams and stability maps of the rotating machinery system by using the Modelica_LinearSystems2 as well as transient simulation results. The presented models show the abilities to design and diagnose rotating machinery systems.

\section{References}

Marcus Baur, Martin Otter and Bernhard Thiele. Modelica Libraries for Linear Control Systems. Proceedings of the 7th International Modelica Conference, 2009. doi: 10.3384/ecp09430068.

Yukio Hori and Koji Kato. Studies on tribology. Proceedings of the Japan Academy, Series B, 84(8): 287-320, 2008.

Tatsuro Ishibashi, Han Bing and Tadao Kawai. Rotating Machinery Library for Diagnosis. Proceedings of the 12th International Modelica Conference, 2017. doi:10.3384/ecp17132381.

Yukio Ishida and Toshio Yamamoto. Linear and Nonlinear Rotordynamics: A Modern Treatment with Applications, 2nd Edition. Wiley-VCH, 2012.

Osami Matsushita, Masato Tanaka, Hiroshi Kanki, Masao Kobayashi and Patrick Keogh. Vibrations of Rotating Machinery. Springer Japan. 2017. doi: 10.1007/978-4-43155456-1.

Martin Otter. The LinearSystems library for continuous and discrete control systems. Proceedings of the 5th International Modelica Conference, 2006. URL https://www.modelica.org/events/modelica2006/Proceedin gs/sessions/Session5c1.pdf/view.

Tsuneo Someya. Journal-Bearing Databook. Springer-Verlag Berlin Heidelberg. 1989. doi: 10.1007/978-3-642-52509-4. 